\title{
Factors Coupled the Choice of Clopidogrel, Prasugrel And Ticagrelor in Patients Undergoing Percutaneous Coronary Intervention
}

\author{
Aswathi Ravindran, Shanly Susan Mathew, Anjali Sunny, Aeby George, Kiron SS, S. M Ashraf, and \\ BinooVimal
}

\begin{abstract}
Background:Percutaneous coronary intervention is used in patients whose coronary arteries are narrowed and blood flow to the heart are obstructed. Antiplatelets has a major role in patients who have undergone PCI. Selection of antiplatelets is very important and study reveals about the factors associated with the selection of antiplatelet agents, thereby providing maximum therapeutic outcome. Objective: To determine the factors influencing the selection of antiplatelet agents in patients undergoing PCI.

Materials And Methods: A prospective observational study was conducted on antiplatelet agents by carefully analyzing the case records of patients who were selected as the study population. For collection of data, a data collection form was prepared. At the end of the study regression analysis was done to find out the factors influencing selection of antiplatelet agents.

Results: 350 patients were followed, $71.4 \%$ males and 28.6 females. Of the 250 male patients 67-SVD, 99- DVD and 84TVD; whereas in females 29-SVD, 36- DVD and 35- TVD. There was a statistically significant correlation between hypertension $(p=0.029)$, body weight $(p=0.001)$, number of stent implanted $(p=0.009)$ and diameter of stent $(p=0.022)$ with drug. The prevalence of $C A D$ was more in the age group 51-75 years and clopidogrel was the most preferred. Prasugrel was not used in patients greater than 75 years because of major bleeding. None of the patients with renal dysfunction were treated with ticagrelor. Conclusion: Hypertension, bodyweight, number of stent implanted, and diameter of stent were significantly associated with the selection of antiplatelet agents in patients undergoing PTCA.
\end{abstract}

Index Terms-Antiplatelets, ACS, DAPT, myocardial infarction, PCI.

\section{INTRODUCTION}

Percutaneous coronary intervention (formerly known as angioplasty with stent) is a non-surgical procedure that uses a catheter to place a small structure called stent to open up blood vessels in the heart that have been narrowed by plaque buildup, a condition known as

Published on December 25, 2019.

Authors are with the Government Medical College, Kannur, India.

(e-mail: aswathiravindran961@gmail.com) atherosclerosis. [1] This procedure is used in patients whose coronary arteries are narrowed and blood flow to the heart are obstructed. ${ }^{[2]}$ Stent is a wire mesh tube made of metal, plastic or a specialized fabric (stent graphs). Different types of stents are available, bare metal stents, drug iluting stents, a bioabsorbable stent, dual therapy stent (which is the combination of both drug and bioengineered stent). ${ }^{[3]}$

Present study deals with three major antiplateletsclopidogrel, prasugrel and ticagrelor. Clopidogrel is the most frequently utilized P2Y12 inhibitor. Clopidogrel is a prodrug that is metabolized to an active form by the cytochrome (CYP) 450 enzyme system in the liver. ${ }^{[4]}$ Prasugrel is a thienopyridine adenosine diphosphate (ADP) receptor inhibitor that inhibits platelet aggregation. It has been shown to reduce new and recurrent MIs. The loading dose is $60 \mathrm{mg}$ orally given once, and the maintenance dosage is $10 \mathrm{mg}$ /day orally (given with aspirin 75-325mg/day). Prasugrel is indicated for reducing thrombotic cardiovascular events (including stent thrombosis) in patients with an ACS that is managed with PCI. It is used specifically for unstable angina or NSTEMI or for acute STEMI that is managed with primary or delayed PCI. ${ }^{5]}$ Ticagrelor, a cyclopentyltriazolo-pyrimidine, is an oral $\mathrm{P} 2 \mathrm{Y} 12$ receptor antagonist that reversibly inhibits platelets. It does not require hepatic bioactivation, because it is an active drug. ${ }^{[6]}$

The study helps to identify the rationale behind the choice of antiplatelet agents for various risk factors and also to compare the difference in patient characteristics across the three drug groups in order to provide additional insight into the use of these drugs and better therapeutic outcome. Antiplatelet medications are high risk drugs which have very small therapeutic window. Too much of a drug can cause bleeding and too little may lead to therapeutical failure (clotting). Hence it is vital to address high risk antiplatelet drugs, carefully asses and closely monitor patients receiving these, to ensure full benefits of antiplatelet therapy and to minimize potential harm. 


\section{MethodolOGY}

This is a prospective observational study done by carefully analyzing the case records of IP patients who were selected as the study population. The study was conducted in SahakarnaHridayalaya, Government Medical College, Kannur. It is a multi-specialty tertiary care hospital of 1200 beds. Study was conducted for a period of 6 months from December 2017 to june 2018. Minimum sample size is 175. All Patients underwent angioplasty were included and patients with Less than 25 years and those with septicemia and cardiogenic shock were excluded from the study. For collection of data, a data collection form was prepared and collected data were entered into a MS Word excel format. It includes various parameters like patient demographics, laboratory data, and comorbid conditions such as Diabetes mellitus, hypertension, dyslipidemia, renal failure, thyroid abnormalities etc. which helps us to determine the most commonly used antiplatelets in various risk conditions and the factors associated with the selection of these antiplatelet agents. The data was evaluated at the end of the study period and proper statistical analysis was done.

\section{RESULT AND DISCUSSION}

A total of 350 patients were followed of which 250 (71.4\%) were males and $100(28.6 \%)$ were females. Of the 250 male patients 67 were with SVD, 99 with DVD and 84 patients with TVD; whereas in females 29 patients had SVD, 36 had DVD and 35 had TVD.

Among the 350 subjects, 157 (44.85\%) patients were treated with clopidogrel, $92(26.28 \%)$ with prasugrel and $101(28.85 \%)$ with ticagrelor

A. Distribution of sample based on age group and drug use

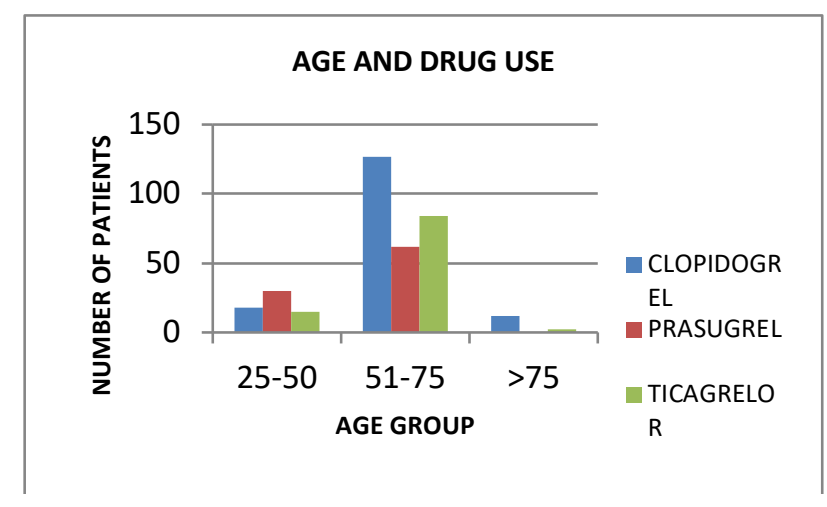

Figure -1:Distribution of sample based on age group and drug use
While comparing all the 3 age groups and drug used it was observed that majority of the patient comes under 5175 years, of which clopidogrel $(127 ; 80.89 \%)$ was the most preferred antiplatelet, followed by ticagrelor $(84 ; 83.16 \%)$ and prasugrel $(62 ; 67.39 \%)$. This is in line with the study by UpendraKaul, Jagdish C Mohan et.al which suggest that clopidogrel is still the preferred drug in those with low ischemic burden and high bleeding risk. Special situations are in those patients who have received thrombolysis or those with deranged renal function and very elderly people. ${ }^{[7]}$

In the age groups 25-50 the most frequently used drug was prasugrel $(30 ; 32.60 \%)$, followed by clopidogrel $(18 ; 11.46 \%)$ and ticagrelor $(15 ; 14.85 \%)$. Similarly, according to UpendraKaul, Jagdish C Mohan et.al prasugrel is best suitable for relatively young patients with ACS and diabetes and especially in those who have once experienced stent thrombosis or recurrence of ACS on clopidogrel therapy. ${ }^{[7]}$

Study also shows that in patients older than 75years prasugrel was not used. This is in line with the study done by Marchini, Julio, et al, in which prasugrel is not recommended in patients older than 75 years due to bleeding risk, except in high risk situations such as diabetes or a prior heart attack. ${ }^{[8]} 12(7.64 \%)$ patients were given clopidogrel and $2(1.98 \%)$ patients treated with ticagrelor.

TABLE-I: CORRELATION BETWEEN AGE AND DIFFERENT VARIABLES

\begin{tabular}{|c|c|}
\hline VARIABLES & P VALUE \\
\hline BODY WEIGHT & 0.001 \\
\hline SYSTOLIC BP & 0.037 \\
\hline DIASTOLIC BP & 0.575 \\
\hline SERUM UREA & 0.038 \\
\hline SERUM CREATININE & 0.417 \\
\hline HEMOGLOBIN & 0.000 \\
\hline PLATELET & 0.213 \\
\hline
\end{tabular}


Here there is a highly significant correlation between age and hemoglobin (0.000); similarly, in the study by Hawkins, WW Speck et.al, it was found that $\mathrm{Hb}$ values in younger men (20-60 years) were typically $14.5 \mathrm{~g}$ to $15 \mathrm{~g}$ per $\mathrm{dL}$, which markedly decreased to an average of 12.4 in men between 80 and 90 years of age.

\section{B. Distribution of sample according to comorbidities}

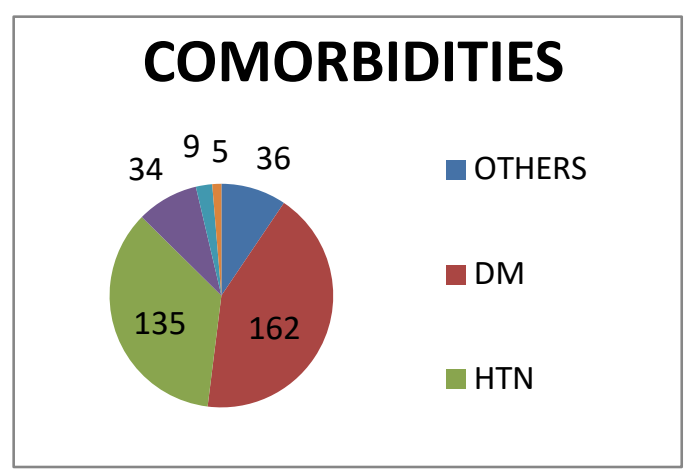

Figure-2: Distribution of sample according to comorbidities

The Important comorbidities coexisting with CAD in this study were DM (46.28\%), HTN (38.57\%), dyslipidemia $(9.71 \%)$ and others $(10.28 \%)$ followed by thyroid (2.57\%)and renal dysfunction (1.42\%).

Others include deepvein thrombosis (DVT), dyselectrolemia, cerebrovascular accident (CVA), COPD, urinary bladder tumor, pulmonary veno occlusive disease and seizure disorder, urinary tract infection, asthma, anaemia, psoriatic arthropathy etc

\section{Drug given in different comorbidities}

While evaluating the pattern of comorbidities and drugs used clopidogrel was mostly prescribed in all conditions. This may be attributed to the fact that clopidogrel is the most cost-effective drug for patients with peripheral vascular disease(PVD) and also it is not associated with serious adverse bleeding complications as suggested by $\mathrm{S}$ Banerjee et.al et.al. [9] Of the 135 patients with hypertension, $71(52.59 \%)$ were treated with clopidogrel, $38(28.14 \%)$ patients with ticagrelor and $26(19.25 \%)$ patients with prasugrel. DM is an independent risk factor for CAD and the most significant one. Anna Norhammar et.al also stated that DM remained an independent and important risk factor for death and MI in the invasive group. Unstable CAD is linked to a substantially increased mortality and morbidity among diabetes patients. ${ }^{[10]}$ Among the diabetic population 65 (40.12\%) patients were prescribed with clopidogrel, 51 (31.48\%) with ticagrelor and 46 (28.39\%) with prasugrel.
In patients with dyslipidemia clopidogrel (44.11\%) was preferred over prasugrel $(32.35 \%)$ and ticagrelor $(23.52 \%)$. Also, in case of thyroid disorders clopidogrel was used in 7 patients $(77.77 \%)$ whereas prasugrel and ticagrelor were used in a single case (11.11\%).

Whereas in renal problems none of the patients were treated with ticagrelor. A. J.VanVuren,B.et.al also documented that renal function deterioration is a frequent side effect of ticagrelor, especially in patients of age greater than 75 or with preexistent mild renal failure. ${ }^{[11]}$ Clopidogrel was used in $4(80 \%)$ patients and prasugrel in $1(20 \%)$ patient.

While in other comorbid conditions drug use follows the order: clopidogrel $(58.33 \%)$, prasugrel $(16.66 \%)$, ticagrelor (25\%).

Statistical value shows $(p=0.029, \quad \mathrm{CI}=0.026-0.035)$ selection of antiplatelets and hypertension have significant statistical relationship.

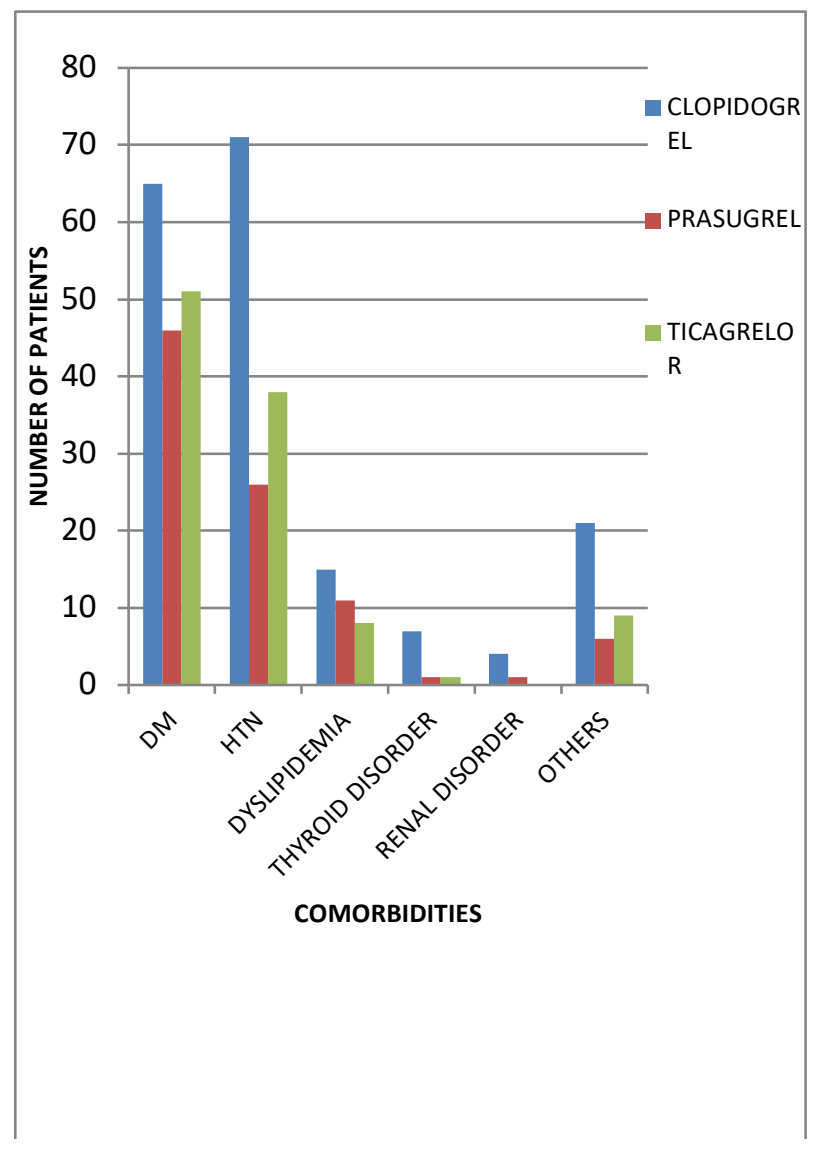

Figure-3: Drug given in different comorbidities 
TABLE-II: CORRELATION BETWEEN DRUG AND COMORBIDITIES

\begin{tabular}{|c|c|}
\hline CO-MORBIDITIES & P VALUE \\
\hline DIABETES MELLITUS & 0.255 \\
\hline HYPERTENSION & 0.029 \\
\hline DYSLIPIDEMIA & 0.711 \\
\hline THYROID ABNORMALITY & 0.132 \\
\hline RENAL DYSFUNCTION & 0.381 \\
\hline OTHERS & 0.337 \\
\hline
\end{tabular}

Statistical value shows $(p=0.029, \quad \mathrm{CI}=0.026-0.035)$ selection of antiplatelets and hypertension have significant statistical relationship. $\mathrm{P}$ value of conditions except hypertension is greater than 0.05 and are not statistically significant.

Spearman's correlation shows $\mathrm{p}$ value $=0.001$, indicating that body weight is a factor influencing the drug choice. These findings can be supported by a study conducted by Julio Marchini, et.al in which prasugrel should not be used in patients with low body weight. ${ }^{[8]}$ Another study conducted by Henrik Wagner, et.al concludes that higher body weight patients on clopidogrelmaintanence therapy have lower active metabolic concentration, lower levels of platelet inhibition and higher rates of poor responders than low body weight patients. [12] The product profile for ticagrelor recommend that no dose adjustment is necessary for ticagrelor based on weight. ${ }^{[13]}$

Body weight of our sample shows a mean value of 63.59 with a standard deviation of \pm 9.309 . systolic and diastolic BP ranges from $125.97 \pm 19.108$ and $78.60 \pm 9.71$.

\section{CAD and Drug}

Patients who were diagnosed to have $\mathrm{CAD}$ were categorized in to SVD, DVD and TVD. Of the total 96 patients diagnosed with SVD clopidogrel was used in $45(46.8 \%)$ patients followed by prasugrel in $28(29.16 \%)$ patients and ticagrelor in 23(23.95\%) patients.

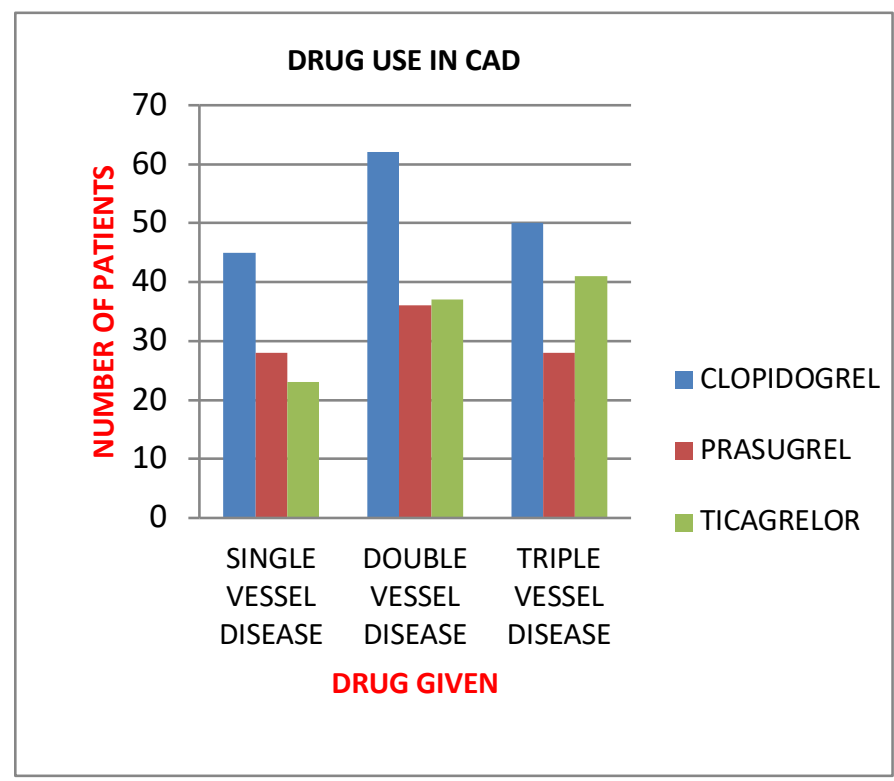

Figure-4: Drug use in CAD

DVD was diagnosed in 135 patients, of these 62 (45.9\%) were treated with clopidogrel, $37(27.4 \%)$ with ticagrelor and $36(26.6 \%)$ patients with prasugrel.

119 patients were diagnosed to have TVD, of which $50(42.01 \%)$ patients were treated with clopidogrel, followed by $41(34.45 \%)$ with ticagrelor and $28(23.52 \%)$ with prasugrel. Clopidogrel was most commonly used to treat patients with SVD, DVD and TVD.

Here there is no statistically significant relationship between selection of drugs and CAD as per Pearson chi square $(\mathrm{p}=0.528)$

\section{E. Number of stent and drug}

In patients implanted with single stent clopidogrel, prasugrel and ticagrelor use were as follows: clopidogrel (104; 51.23\%), ticagrelor $(52 ; 25.61 \%)$, prasugrel $(47$; $23.15 \%)$. Similarly,clopidogrel $(47 ; 40.86 \%)$ was used more in patients undergone implantation with 2 stents whereas prasugrel and ticagrelor were used equally (34; $29.56 \%$ ). While in case of 3 stent implantation ticagrelor $(15 ; 46.87 \%)$ use was followed by prasugrel $(11 ; 34.37 \%)$ and clopidogrel $(6 ; 18.75 \%)$. Boris Schnorbus, et.al reported that the beneficial effects of clopidogrel are lost upon prolonged treatment and acutely clopidogrel does not prevent stent induced vascular dysfunction, in patients undergoing coronary stenting. ${ }^{[14]}$ 


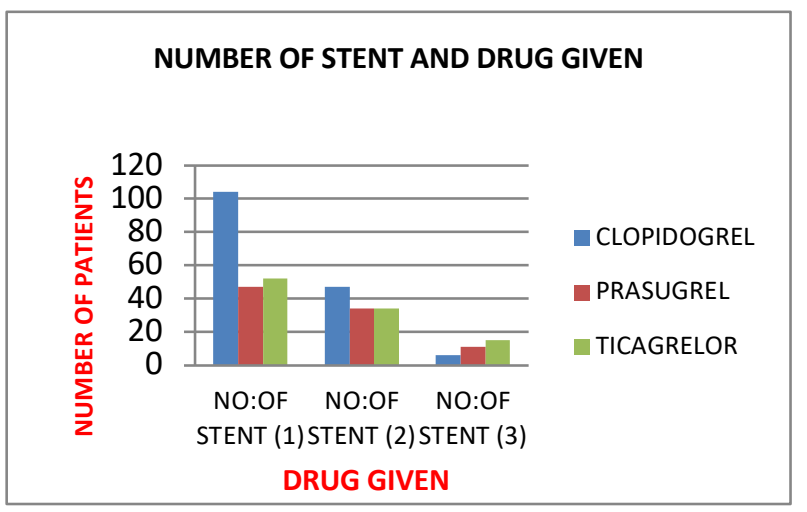

Figure-5: Drug given according to stent used

Correlation statistics was performed for assessing relationship between number of stent and drugs and $p$ value $=0.009$ which indicates that the selection of antiplatelets and number of stents used have significant relationship.

\section{CONCLUSION}

According to the study, prevalence of CAD was more in the age group 51-75 years and clopidogrel was the most preferred antiplatelet. Results of the study also shows that prasugrel was not used in patients greater than 75 years because of major bleeding. None of the patients with renal dysfunction were treated with ticagrelor. Findings also suggest that there is a statistically significant correlation between the following factors and selection of antiplatelets: hypertension $(p=0.029), \quad$ body weight $(p=0.001)$, number of stent implanted $(p=0.009)$ and diameter of the stent $(p=0.022)$. Hence these are some of the factors associated with the choice of antiplatelet agents in patients undergoing PTCA. These data can be used for clinical decision making and different epidemiological studies for the improvement of healthcare.

\section{ACKNOWLEDGEMENT}

The authors thank doctors and staff of Government Medical College, Kannur for providing required facilities to carry out this work.

\section{REFERENCES}

[1] Percutaneous coronary intervention (PCI or angioplasty with stent) [available from internet] https://www.heartandstroke.com

[2] Percutaneous coronary intervention [available from internet] https://www.percutaneouscoronaryintervention.com

[3] Stent, American Heart Association [available from internet] https://www.heart.org.com

[4] Ellis KJ; Stouffer GA; McLeod HL; Lee CR. Clopidogrel pharmacogenomics and risk of inadequate platelet inhibition: US
FDA recommendations. Pharmacogenomics. 2009; 10(11):1799817.

[5] https://reference.medscape.com/drug/effient-prasugrel

[6] Ticagrelor [available from internet] https://en.m.wikipedia.org/wiki/Ticagrelor

[7] Choice of antithrombotics in acute... (PDF Download Available) [Internet]. [cited 2018 Jun 2]. Available from: https://www.researchgate.net/publication/297735901 Choice of antithrombotics in acute coronary syndrome A balance of efficacy versus safety

[8] Marchini J, Morrow D, Resnic F, Manica A, Kirshenbaum J, Cannon C, et al. An Algorithm for use of Prasugrel (Effient) in Patients Undergoing Cardiac Catheterization and Percutaneous Coronary Intervention.CritPathwCardiol. 2010 Dec;9(4):192-8.

[9] Clopidogrel versus Other Antiplatelet Agents for Secondary Prevention of Vascular Events in Adults with Acute Coronary Syndrome or Peripheral Vascular Disease: Clinical and CostEffectiveness Analyses | CADTH.ca [Internet]. [cited 2018 Jun 2]. Available from: https://www.cadth.ca/clopidogrel-versusother-antiplatelet-agents-for-secondary-prevention-vascularevents-adults

[10] Norhammar A, Diderholm E, Lagerqvist B, Lindahl B, Rydén L, Wallentin L. Diabetes mellitus: the major risk factor in unstable coronary artery disease even after consideration of the extent of coronary artery disease and benefits of revascularization. J Am CollCardiol. 2004;43(4):585-591

[11] Van Vuren AJ, De Jong B, Bootsma HPR, Van der Veen MJ, Feith GW. Ticagrelor-induced renal failure leading to statininduced rhabdomyolysis.Neth J Med. 2015;73(3):136-38.

[12] Wagner H, Angiolillo DJ, Ten Berg JM, Bergmeijer TO, Jakubowski JA, Small DS, et al. Higher body weight patients on clopidogrel maintenance therapy have lower active metabolite concentrations, lower levels of platelet inhibition, and higher rates of poor responders than low body weight patients. J Thromb Thrombolysis. 2014;38(2):127-36.

[13] Brilinta ${ }^{\mathrm{TM}}$ (ticagrelor) Tablets. Pharm Ther. 2012 Apr;37(4 section 2):4-18

[14] Schnorbus B, Daiber A, Jurk K, Warnke S, König J, Krahn U, et al. Effects of clopidogrel, prasugrel and ticagrelor on endothelial function, inflammatory and oxidative stress parameters and platelet function in patients undergoing coronary artery stenting for an acute coronary syndrome. A randomised, prospective, controlled study.BMJ Open. 2014;4(5): e005268 


\section{AUTHORS:-}

AswathiRavindran, Shanly Susan Mathew, Anjali Sunny, Aeby GeorgePHARM D Interns at government Medical College, Kannur. DrKiron SS, Professor, Department of Pharmacy Practise, Government Medical, College, Kannur. He has completed his B Pharm, M Pharm(Pharmacy Practice) and Ph.D. Dr S.M Ashraf, Interventional Cardiologist, SahakaranaHridayalaya, Government Medical College, Kannur. Has 10 years experience in Interventional Cardiology and is also an experienced teacher for the DM course. Holds MBBS, MD and DM degrees. He is a fellow of the society for cardiovascular angiography and interventions(FSCAI), as well as a fellow of the European society of cardiologist (FESC). 
EJMED, European Journal of Medical and Health Sciences Vol. 1, No. 5, December 2019 